\title{
Kommunikation mit Partnern nach Cochlea Implantat
}

\section{Patienteninformation}

\section{Die Bedeutung gelingender Kommunikation}

Durch die veränderte Hörempfindung einer CI-tragenden Person werden Sprachlaute, Wortbetonungen oder die Satzmelodie, in geringerem Ausmaß gehört, so dass Gesprächsinformationen erschwert wahrgenommen werden. Eine erschwerte Kommunikation kann weitreichende Folgen haben und zu Resignation, Rückzug und sozialer Isolierung führen. Demgegenüber hat eine gelingende Kommunikation großen positiven Einfluss auf das Leben mit der Hörbeeinträchtigung.

\section{Wie kann der Partner unterstützen? $\nabla$}

Angemessenes Sprechverhalten

„Klar“ zu sprechen heißt nicht automatisch, lauter zu sprechen. Eine deutlich zu verstehende Sprache wird am besten durch ein langsameres Sprechtempo erzielt. Langsameres Sprechen bewirkt eine bewusste und klare Aussprache. Gleichzeitig sollte nicht „überartikuliert“ werden. Eine Überartikulation bewirkt eine Verzerrung des Gesprochenen und kann sogar zu erschwertem Verstehen führen. Hilfreich ist das Setzen von Pausen nach wichtigen Wörtern oder am Satzende. Betonungen können durch leichtes Anheben der Stimmlage unterstützt werden.

\section{Blickkontakt sicherstellen und Mundbild nicht verdecken}

Personen mit Cl erhalten wichtige Zusatzinformationen durch die Beobachtung von Gesicht, Sprechbewegungen und Mimik. Es ist wichtig, den Blickkontakt zu halten und das Gesicht nicht zu verdecken. Sprechen mit Essen im Mund und blendendes Licht sollten vermieden werden. Glänzende Gegenstände wie Spiegel und weiße Tischoberflächen können blenden und störend wirken. Sie schränken das Erkennen des Gesichtes ein. Optimal ist, wenn die Person mit CI die Lichtquelle im Rücken hat, um das Gesicht des Gesprächspartners gut erkennen zu können.

\section{Einfühlungsvermögen}

Die Gesprächspartner sollten sich der schwierigen Aufgabe bewusst sein, Sprache mit herabgesetztem Hörvermögen zu erfassen. Es kostet mehr Anstrengung und Konzentration, Gespräche zu führen, da ein ständiger Prozess des Ergänzens von fehlenden Hörinformationen ausgeglichen werden muss. Geduld und Verständnis sind daher eine wichtige Unterstützung, um Stress und Angst zu vermeiden.

\section{Gestalten sprachlicher Äußerungen} Präzise Informationen ohne Unklarheiten oder Doppeldeutigkeiten helfen, um nicht zu verwirren oder unnötige Konzentration abzuverlangen. Im Gesprächsverlauf sollte aufmerksam verfolgt werden, ob das gesagte verstanden wurde.

\section{Gesprächsabbrüche}

Wird der Gesprächsverlauf unterbrochen oder entsteht eine Missverstehens-Situation, so kann man verschiedene Reparaturstrategien verwenden:

- Umformulieren des Gesagten,

- Hervorheben von Schlüsselwörtern,

- Verkürzen einer Aussage,

- Unterstützen durch Gestik oder

- Aufschreiben von Informationen, z. B. eines vereinbarten Termins.

Der Beginn des Sprechens kann durch ein vereinbartes Zeichen angekündigt werden, wie z. B. durch Anheben des Zeigefingers.

\section{Erschwerte Gesprächssituationen}

Erschwerte Kommunikationsumgebungen sind Situationen in geräuschvoller Umgebung (z. B. Bus, Geschäfte, Feier, Restaurant). Um Gespräche in solchen Situationen zu unterstützen, kann z. B. ein Restaurant im Vorfeld anhand von Merkmalen zur Raumakustik und zum Störschall ausgewählt werden. Bedeutsam sind:

\author{
- Lautstärke der Musik, \\ - offene Küche, \\ - Gebläse oder \\ - schallschluckende Stoffe.
}

Empfehlenswert ist es, entfernt von der Tür, mit guter Sicht auf alle Gesprächsteilnehmer, zu sitzen. In Gruppengesprächen kann eine Person als Stichwortgeber vereinbart werden. Dieser weist auf Sprecher- und Themenwechsel hin, so dass der Einstieg in eine Äußerung und das Verstehen des Gesprächsverlaufs unterstützt werden.

\section{Fazit}

Ein Gespräch wird von allen beteiligten Personen gestaltet. Somit leisten Kommunikationspartner einen wichtigen Beitrag für das Gelingen von Gesprächen. Wichtig sind eine unterstützende, verständnisvolle Grundeinstellung gegenüber der Lebenssituation mit $\mathrm{CI}$ und gleichzeitig das Wissen, worin die positive Unterstützung liegen kann. Grundlegend ist der Austausch zwischen den Kommunikationspartnern, wie die Hilfen erlebt und gewünscht werden.

\section{Sybille Seybold, Oldenburg}

Weiterführende Literatur

Hickson L, Worrall L, Scarinci N. Active Communication Education Program for Older People with Hearing Impairment. Geelong: Speechmark; 2007

Manchaiah VKC, Stephens D, Lunner T. Communication Partners' Journey through Their Partner's Hearing Impairment. Int J Otolaryngol. 2013;2013:707-910

Tye-Murray N. Foundations of Aural Rehabilitation - Children, Adults and Their Family Members. 4th Ed, Stamford: Cengage Learning; 2015

überreicht durch Thieme und 